\section{Dual mechanisms in passive avoidance: I}

ROBERT J. 'BLANCHARD and. D. CAROLINE BLANCHARD, University of Hawaii, Honolulu, Hawaii 96822

Female rats received shock through objects varying in discriminability. Poorly discriminable objects elicited rapid avoidance acquisition, with suppression of activity and subsequent avoidance of the shock chamber (increased entry latencies). Highly discriminable shock objects also elicited rapid acquisition of avoidance, but without activity suppression or chamber avoidance. This pattern of findings suggests dual mechanismṣ for passive avoidance, with discriminated avoidance underlying failure to contact highly discriminable shock objects, and response suppression (immobility) underlying avoidance of poorly discriminable sources of threat.

Blanchard \& Blanchard (1969a) have suggested that immobility reactions elicited by situational stimuli previously paired with shock may greatly facilitate passive avoidance by decreasing activity in the shock situation. Since such immobility reactions do not appear to be elicited by discrete, specific stimuli associated with shock (Blanchard \& Blanchard, 1969b), it might be expected that immobility effects in passive avoidance are a function of the discriminability of the object or area through which shock is given. That is, when shock is given through an easily discriminated object, passive avoidance performance may largely reflect fear of that object without concomitant activity reduction in the shock situation. However, if the source of the shock cannot be discriminated by the $S$, the entire situation may elicit fear, resulting in immobility in the shock situation.

\section{SUBJECTS}

The Ss were 40 naive female albino rats, 80-85 days of age, from the colony maintained by the University : of Hawaii Department of Psychology.

\section{APPARATUS}

The test situation was a $20 \times 20 \times 12$ in. wooden chamber with $1 / 4$-in. stainless stee bars as flooring and one clear Plexiglas wall to permit observation. The remaining walls and the ceiling of the chamber were painted medium flat gray. A $60-W$ bulb suspended $20 \mathrm{in}$. over an 8-in.-square Plexiglas insert in the center of the ceiling provided illumination. This chamber floor was divided into nine squares by a marked sheet suspended $1 / 2$ in. under the steel grids. The chamber was located in a sound-attenuating room, with its Plexiglas side facing a one-way-vision mirror. A $9 \times 5$ in. unpainted plywood startbox was connected to the test chamber by a black Plexiglas guillotine door.

The same chamber was used with each shock object. For the high discriminability condition, the shock object was a $2 \times 3 \frac{1}{2} \times 1 \frac{1}{2}$ in. sheet-metal box, attached to the center of one wall of the chamber: This box, which projected into the chamber, was located $1 \frac{11}{2}$ in. above the grid bars. On three sides of the box, circular holes, $3 / 4$ in. in diam and with a translucent Plexiglas backing, were cut in the sheet metal. A flashing, 6-W bulb inside the box was visible through each of these holes. The box was attached to the chamber wall by a socket arrangement, and could be quickly removed and replaced.

For the intermediate discriminability condition, a $2 \times 31 / 2$ in. platform, constructed of $1 / 4$-in. aluminum glued to a 1/8-in. Plexiglas sheet of the same dimensions was fixed directly on the grids, under the position occupied by the box in the high-discriminability condition.

For the low-discriminability condition, a $2 \times 3 \frac{1}{2}$ in. portion of the grid flooring served as the shock object.-This portion consisted of segments of five of the central grids running from the observation (Plexiglas) wall of the chamber, back to the wall in which the shock objects were centered. These grids were cut 3\%/ in. from the back wall and the cut ends force-fitted into the ends of clear Plexiglas cylinders, $3 / 4$ in. long and $1 / 3$ in. in diam, so that the cut ends of the grids did not touch. Thus, except for the presence of the clear Plexiglas cylinders, the isolated grids were identical to the remaining grid flooring The isolated grids were immediately under the area where the shock box and shock platform were placed during the high- and intermediate-discriminability conditions respectively.

\section{PROCEDURE}

All Ss were individually housed and given free access to food and water throughout the experimental period. The Ss were divided randomly into five groups, with eight .Ss per group. These groups were: grid shock (GS), grid control (GC), platform shock (PS), box shock (BS), and box control (BC). The Ss were run according to a predetermined random order between 5 and 12 p.m. daily.

In the first session, the $S$.was brought to the experimental room and placed in the test chamber on a square adjacent to the Plexiglas observation wall. For $\mathrm{BS}$ and $\mathrm{BC}$ Ss. the test chamber contained the box with flashing light. For the PS group, the chamber contained the sheet-metal platform. For GS and GC groups, the test chamber contained only the grid flooring with electrically isolated segments. For all shocked groups; the shock: objects were electrified $(3.0 \mathrm{~mA})$ before the Ss were placed in the chamber. The $\mathrm{BC}$ and $\mathrm{GC}$ groups received no shock.

Contacts of the nonelectrified shock objects (i.e., during Retests 1 and 2 for all Ss and during the first test session for the nonshocked Ss) were measured by a Grason-Stadler drinkometer circuit. Shocks received by the shocked groups during the first test session were monitored by a neon indicating tube coupled with a photoelectric pen recording system. The number of squares entered before contact with the shock object and squares entered during each minute of the 5-min postcontact test period were recorded by an $E$ who observed the $S$ through the one-way-vision mirror. After the test period, the Ss were replaced in their home cages.

Thirty minutes after the first test period, each $S$ was retested. In the retest, the appropriate shock object for each $\mathrm{S}$ was placed in the chamber, but no shocks were given to any $S$. The Ss were placed in the startbox, and, after $15 \mathrm{sec}$, the guillotine door was raised, permitting entry into the test chamber. Latency of entry into the test chamber was recorded. During each minute of the 5-min period following entry into the test chamber, the number of shock-object touches and the squares entered were recorded. A second retest, identical in procedure to the first retest, was given $24 \mathrm{~h}$ after initial training.

\section{RESULTS}

Table 1 presents the number of shock-object contacts for the control groups in all sessions and for the shocked groups in Retests 1 and 2. This table also presents the number of shocks received by the shocked groups in the initial test session. Since the distributions for shock-object contacts for the shocked groups and for the number of shocks tended to be skewed, Mann-Whitney $\mathrm{U}$ tests were used in statistical analyses of group differences. Although differences in the number of shocks received for the GS, PS, and BS groups were not large, they were quite consistent. Statistical analysis indicated that the GS Ss received reliably more shocks than the PS $(U=11.5$, $\mathrm{p}<.025)$ or the $\mathrm{BS}^{-}(\mathrm{U}=6, \mathrm{p}<.005) \mathrm{Ss}$. The PS and. BS "groups did not differ reliably $(U=24)$.

In the retest sessions, all shocked groups tended to make fewer shock object contacts than did controls. These experimental-control differences were 
Table 1

Shocks, Shock Object Contacts, and Entry Latencies for All Groups

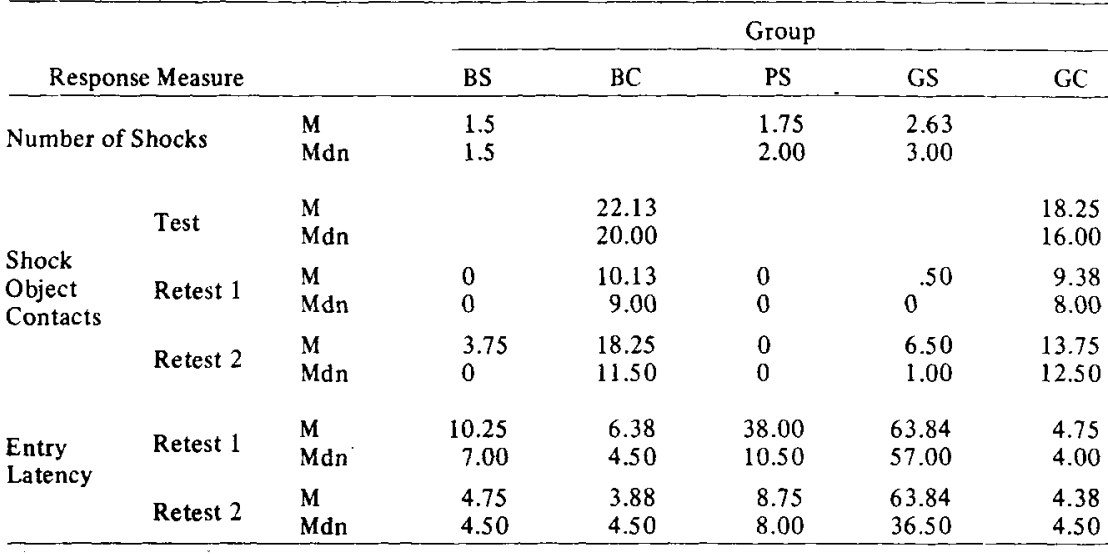

reliable in both the first and second retest sessions (GS-GC, $U=5, p<.001$, and $\mathrm{U}=14.5, \mathrm{p}<.05$, respectively; $\mathrm{BS}-\mathrm{BC}$, $\mathrm{U}=0, \mathrm{p}<.001$ and $\mathrm{U}=7, \mathrm{p}<.005$, respectively). In both retest sessions, differences of the BS, PS, and GS groups failed to reach an acceptable level of statistical significance.

Figure 1 presents the mean number of test chamber squares entered for the three test sessions for all groups. In the initial test session, the $B C$ and $B S$ groups were not reliably different on this activity measure, while the GS Ss were reliably less active than their controls $(t=3.47$, $p<.005$; all ts with $14 \mathrm{df}$ ).

In the first retest session, the $B C$ and $B S$ groups' activity difference again failed to reach an acceptable level of significance, while the GS Ss continued to be reliably less active than the $G C$ Ss $(t=3.60$, $\mathrm{p}<.005)$. Although the BS Ss were reliably more active than the GS Ss $(t=3.91, p<.005)$, the PS group's activity scores were not reliably different from those of either the BS or GS groups. As in

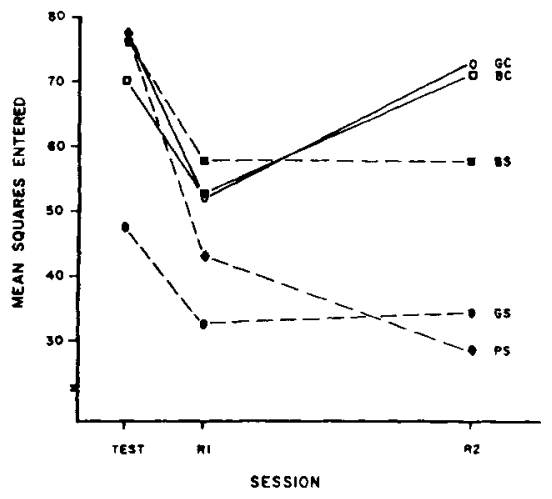

Fig. I Mean squares crossed by each group, during the test and retest sessions. the test and first retest sessions, GS Ss' activity scores in the second retest session group $(t=5.22, p<.005)$. In this second test session, activity for the BS group was reliably greater than that of the GS $(t=2.13, p<.05)$ or the PS $(t=2.73$, $\mathrm{p}<.01$ ) groups.

Latency to enter the test chamber was measured for all groups during the two presented in Table 1. Comparison of the mean and median values for each of the groups indicates that the entry latency distributions for the shocked groups tended to be skewed. Therefore, as in the case of the shock-object contact scores, Mann-Whitney U tests were used to determine the statistical significance of differences between groups. In both retest sessions, the two control groups and the BS group tended to have similar entry latencies. In neither retest session did the latency difference of the $\mathrm{BC}$ and $\mathrm{BS}$ groups approach an acceptable level of significance. Also, in both retest sessions, the PS group's latency scores tended to be slightly higher than the BS group's latencies. The PS group's latencies were not reliably different from those of the BS there was a reliable latency difference between these two groups in the second retest session $(U=13.0, p<.025)$. As indicated in Table 1, latencies for the GS group were much higher than those of any other group in both retest sessions. In the first retest session, the GS latency scores were reliably greater than those of the GC $(\mathrm{U}=2, \mathrm{p}<.001)$ or the BS $(\mathrm{U}=7$, $\mathrm{p}<.005)$ groups. Latency differences between the GS and PS groups just failed to reach an acceptable level of significance $(\mathrm{U}=16, \mathrm{p}>.05)$. This pattern was were reliably less than those of the GC retest sessions. These latency scores are group in the first test session. However, repeated in the second retest session, with reliable latency differences between the GS and $\mathrm{GC}(\mathrm{U}=7.5, \mathrm{p}<.01)$ and between the GS and BS $(U=8.5, p<.01)$ groups. However, in the second test session, the GS group's latency scores weie also reliably greater than those of the PS group $(\mathrm{U}=14.5, \mathrm{p}<.05)$.

\section{DISCUSSION}

The results of the present study clearly indicate that shock objects differing in discriminability elicit different behavioral patterns following shock. The findings of consistent decrements in the number of shock-object contacts for all experimental groups, in all test and retest sessions, indicate that both high- and low-discriminable shock objects may quickly come to elicit avoidance. However, other behavioral indices in the test situation suggest the mechanisms associated with behavioral avoidance may vary with the discriminability of the object through which shock was given.

First, the Ss shocked through an isolated portion of the grids were consistently less active than either their controls or the BS Ss. This finding is fully congruent with a hypothesis that situational fear elicits immobility, as is the finding of an intermediate activity position for the PS group. Also, this hypothesis is supported by the findings of reliably increased test-chamber entry latencies for the GS Ss, with the PS group again tending to fall between the GS and BS groups. On both the activity and entry latency measures, the BS Ss' scores suggest that shock through a highly discriminable object elicits minimal fear of the situation in which shock is given.

Thus, the results of the present study are congruent with a hypothesis that shock-object discriminability influences the patterning of subsequent avoidance. However, this study does permit one alternative interpretation. Since shock-object discriminability was associated with a differential number of test-session shocks, it is possible that the number of shocks, rather than the discriminability of the objects through which shock was given, is the primary determinant of the behavior patterns associated with avoidance.

\section{REFERENCES}

BLANCHARD, R. J., \& BLANCHARD, D. C Crouching as an index of fear. Journal of Comparative \& Physiological Psychology, 1969a, 67, 370-375.

BLANCHARD, R. J., \& BLANCHARD, D. C. Passive and active reactions to fear eliciting stimuli. Journal of Comparative \& Physiological Psychology, 1969b, 68, 129-135. 